\title{
Association of Sarcopenia and Gut Microbiota Composition in Older Patients with Advanced Chronic Kidney Disease, Investigation of the Interactions with Uremic Toxins, Inflammation and Oxidative Stress
}

\author{
Elisabetta Margiotta ${ }^{1}$, Lara Caldiroli ${ }^{1}$, Maria Luisa Callegari ${ }^{2}{ }^{(D}$, Francesco Miragoli ${ }^{2}$, Francesca Zanoni ${ }^{1}$, \\ Silvia Armelloni ${ }^{1}$ D, Vittoria Rizzo ${ }^{3}$, Piergiorgio Messa ${ }^{1,4}$ and Simone Vettoretti ${ }^{1, *(\mathbb{D})}$
}

1 Division of Nephrology, Dialysis and Renal Transplantation, Fondazione IRCCS Cà Granda Ospedale Maggiore Policlinico, 20122 Milano, Italy; elisabetta.margiotta@policlinico.mi.it (E.M.); lara.caldiroli@policlinico.mi.it (L.C.); francescazanoni70@gmail.com (F.Z.); silvia.armelloni@policlinico.mi.it (S.A.); piergiorgio.messa@unimi.it (P.M.)

2 Centro di Ricerche Biotecnologiche, Università Cattolica del Sacro Cuore, 26100 Cremona, Italy; marialuisa.callegari@unicatt.it (M.L.C.); francesco.miragoli@unicatt.it (F.M.)

3 Laboratory Medicine and Clinical Biochemical Analysis, Ospedale San Matteo di Pavia, 27100 Pavia, Italy; v.rizzo@smatteo.pv.it

4 Department of Clinical Sciences and Community Health, University of Milan, 20122 Milan, Italy

* Correspondence: simone.vettoretti@policlinico.mi.it; Tel.: +39-0255-034552

\section{check for}

updates

Citation: Margiotta, E.; Caldiroli, L.; Callegari, M.L.; Miragoli, F.; Zanoni,

F.; Armelloni, S.; Rizzo, V.; Messa, P.; Vettoretti, S. Association of

Sarcopenia and Gut Microbiota

Composition in Older Patients with Advanced Chronic Kidney Disease, Investigation of the Interactions with Uremic Toxins, Inflammation and Oxidative Stress. Toxins 2021, 13, 472. https://doi.org/10.3390/ toxins 13070472

Received: 4 May 2021

Accepted: 2 July 2021

Published: 8 July 2021

Publisher's Note: MDPI stays neutral with regard to jurisdictional claims in published maps and institutional affiliations.

Copyright: (C) 2021 by the authors. Licensee MDPI, Basel, Switzerland. This article is an open access article distributed under the terms and conditions of the Creative Commons Attribution (CC BY) license (https:/ / creativecommons.org/licenses/by/ $4.0 /)$.

\begin{abstract}
Sarcopenia is a prevalent condition in chronic kidney disease (CKD). We determined gut microbiota (gMB) composition in CKD patients with or without sarcopenia. Furthermore, we investigated whether in these patients, there was any association between gMB, uremic toxins, inflammation and oxidative stress. We analyzed gMB composition, uremic toxins (indoxyl sulphate and p-cresyl sulphate), inflammatory cytokines (interleukin 10, tumor necrosis factor $\alpha$, interleukin 6 , interleukin 17, interleukin 12 p70, monocyte chemoattractant protein-1 and fetuin-A) and oxidative stress (malondialdehyde) of 64 elderly CKD patients $\left(10<\mathrm{eGFR}<45 \mathrm{~mL} / \mathrm{min} / 1.73 \mathrm{~m}^{2}\right.$, not on dialysis) categorized as sarcopenic and not-sarcopenic. Sarcopenia was defined according to European Working Group on Sarcopenia in Older People 2 criteria. Sarcopenic patients had a greater abundance of the Micrococcaceae and Verrucomicrobiaceae families and of Megasphaera, Rothia, Veillonella, Akkermansia and Coprobacillus genera. They had a lower abundance of the Gemellaceae and Veillonellaceae families and of Acidaminococcus and Gemella genera. GMB was associated with uremic toxins, inflammatory cytokines and MDA. However, uremic toxins, inflammatory cytokines and MDA were not different in sarcopenic compared with not-sarcopenic individuals, except for interleukin 10, which was higher in not-sarcopenic patients. In older CKD patients, gMB was different in sarcopenic than in not-sarcopenic ones. Several bacterial families and genera were associated with uremic toxins and inflammatory cytokines, although none of these latter substantially different in sarcopenic versus not-sarcopenic patients.
\end{abstract}

Keywords: chronic kidney disease; sarcopenia; gut microbiota; inflammation; oxidative stress

Key Contribution: Gut microbiota composition is different between sarcopenic and not sarcopenic CKD patients and it is correlated with many uremic toxins and inflammatory parameters.

\section{Introduction}

Sarcopenia is defined as a loss of skeletal muscle mass and function. In Chronic Kidney Disease (CKD) patients, sarcopenia is highly prevalent and it is associated with physical disability, low quality of life and increased mortality [1]. In these patients, sarcopenia is determined by a series of complex mechanisms, such as malnutrition, systemic 
inflammation, uremic toxins retention, metabolic acidosis, vitamin D deficiency, insulin resistance and some other hormonal factors often present in CKD patients [2].

In CKD patients, retention of uremic molecules and pro-inflammatory cytokines is the key mechanism leading to oxidative stress and inflammation [3]. In particular, two uremic toxins, indoxyl sulphate (IS) and p-cresyl sulphate (PCs), are produced by proteolytic bacterial fermentation in the gut [4,5] and retained in the serum of CKD patients owing to both increased intestinal production and reduced glomerular filtration and proximal tubular secretion [6-8]. IS has been shown to be associated with aortic calcification and vascular smooth muscle cell proliferation in rats and humans $[6,9,10]$; elevated PCs has been associated with insulin resistance [7] and vascular disease [8]. Both toxins play an important role in inducing muscular atrophy through various mechanisms (e.g., pro-inflammatory cytokines release or activation of some pathways, such as aryl hydrocarbon receptor (AhR), $\mathrm{NF} \kappa \mathrm{B}$ and SRAA) [11]. IS contributes to skeletal muscle loss by inhibiting myoblast proliferation through an enhanced expression of myostatin and atrogin-1 mediated by oxidative stress [11]. Moreover, IS promotes metabolic alterations with consequent mitochondrial dysfunction and suppression of anabolism signaling and ATP production [11]. Finally, IS induces atrophy of myotubes through the production of reactive oxygen species [12]. In patients with end stage renal disease in dialysis, there is a significant inverse association between plasma IS and skeletal muscle mass [11], but this association was not observed in older patients with advanced CKD not yet in dialysis [13].

Pathophysiological pathways by which PCs may influence the onset of sarcopenia are different. In a murine model, Koppe et al. demonstrated that PCs inhibits insulin-stimulated glucose uptake and, by activating ERK kinase, decreases insulin signaling pathways. Moreover, PCs suppresses insulin-induced phosphorylation of Akt, decreasing muscle protein synthesis and inducing protein degradation [7]. Therefore, it is likely that IS and PCs may induce the onset of sarcopenia by interfering with different metabolic pathways.

In addition to the role played by uremic toxins, it has recently been demonstrated that intestinal dysbiosis and gastrointestinal barrier disruption may significantly contribute to sustaining a low-grade systemic inflammation in CKD [11,12]. In CKD patients, gut dysbiosis is characterized by distinctive qualitative changes, such as increased the Enterobacteriaceae and reduced the Lactobacillaceae and Prevotellaceae families [13-15]. In our previous study, in which we compared CKD patients with healthy controls, we found that the former were characterized by increased abundance of the Citrobacter, Anaerotruncus, Coprobacillus genera and Ruminococcus torques species and reduced abundance of saccharolytic and butyrate-producing bacteria (Prevotella, F. prausnitzii, Roseburia) [16].

Furthermore, we observed several relevant differences in the gMB composition between frail and not-frail CKD patients, where frail patients showed higher abundance of the Lactobacillus, Oscillospira, Eggerthella (particularly E. lenta species), Erwinia, Anaerotruncus, Coprobacillus genera and the Coriobacteriaceae and Mogibacteriaceae families [16].

The principal aim of this study was to examine if in older patients with advanced $\mathrm{CKD}$, the gMB composition may differ between sarcopenic and not-sarcopenic individuals. Secondly, we explored whether in these patients, there may be any association of gMB with uremic toxins, inflammatory cytokines and oxidative stress. Finally, we explored the association between these latter substances and sarcopenia in this population.

\section{Results}

\subsection{Population Characteristics}

We evaluated 64 patients with a median age of $80.7 \pm 6.2$ years, mostly males $(69 \%)$. Thirty-seven patients were diabetic. They had a median eGFR of $26 \pm 11 \mathrm{~mL} / \mathrm{min} / 1.73$ $\mathrm{m}^{2}$ and a median BMI of $28.4 \pm 4.7$. Sarcopenia had a prevalence of $28 \%(18 / 64)$. Clinical parameters (age, presence of frailty, BMI, CKD-EPI GFR, MIS and PCR) did not differ between sarcopenic and not-sarcopenic patients, except for BMI, which was higher in the not-sarcopenic ones. (Table 1). 
Table 1. Clinical parameters in sarcopenic and not-sarcopenic individuals.

\begin{tabular}{ccccc}
\hline Variables & $\begin{array}{c}\text { Overall Cohort } \\
(\boldsymbol{n}=\mathbf{6 3})\end{array}$ & $\begin{array}{c}\text { Sarcopenic } \\
(\boldsymbol{n}=\mathbf{1 8})\end{array}$ & $\begin{array}{c}\text { Not Sarcopenic } \\
(\boldsymbol{n}=\mathbf{4 5})\end{array}$ & $\boldsymbol{p}$ \\
\hline Age (years) & $80.7 \pm 6.2$ & $83.1 \pm 5.7$ & $79.7 \pm 6.2$ & 0.0528 \\
\hline Males, $n(\%)$ & $44(69)$ & $16(89)$ & $28(62)$ & 0.12 \\
\hline eGFR $\left(\mathrm{ml} / \mathrm{min} / 1,73 \mathrm{~m}^{2}\right)$ & $26 \pm 11$ & $23.9 \pm 10$ & $25.5 \pm 10$ & 0.5733 \\
\hline Diabetes, $n(\%)$ & $37(59 \%)$ & $11(61 \%)$ & $26(58 \%)$ & 0.75 \\
\hline Frailty, $n(\%)$ & $38(59)$ & $13(72)$ & $24(53)$ & 0.16 \\
\hline $\mathrm{BMI}\left(\mathrm{kg} / \mathrm{m}^{2}\right)$ & $28.4 \pm 4.7$ & $25.5 \pm 2.6$ & $29.3 \pm 4.8$ & 0.025 \\
\hline $\mathrm{MIS}$ & $6 \pm 4.7$ & $6.7 \pm 4.2$ & $5.7 \pm 4.9$ & 0.48 \\
\hline $\mathrm{CRP},(\mathrm{mg} / \mathrm{dl})$ & $0.3 \pm 0.5$ & $0.43 \pm 0.9$ & $0.28 \pm 0.22$ & 0.20 \\
\hline $\mathrm{HCO}{ }^{-}$ & $24.0 \pm 3.5$ & $24.0 \pm 3.6$ & $23.9 \pm 2.9$ & 0.93 \\
\hline
\end{tabular}

eGFR, estimated glomerular filtration rate; BMI, Body Mass Index; MIS, Malnutrition Inflammation Score; CRP, C-reactive protein; $\mathrm{HCO}_{3}{ }^{-}$, serum bicarbonate.

\subsection{Sarcopenia and gMB Composition}

We evaluated the gMB composition by examining a total of 8 phyla, 34 families and 52 genera. The statistically different families and genera between $S$ and NS patients are shown in Figures 1 and 2, while full analyses are reported in Supplementary Materials (Figures S1 and S2, Tables S1 and S2). All data refer to direct correlations without any adjustment for other covariates.

In the sarcopenic group, there was a greater abundance of the Micrococcaceae $(\mathrm{FDR}=0.012$ ) and Verrucomicrobiaceae (FDR $=0.012)$ families and a lower abundance of the Veillonellaceae $(\mathrm{FDR}=0.012)$ and Gemellaceae $(\mathrm{FDR}=0.042)$ families (Figure 1$)$. Regarding genera, sarcopenic subjects had greater abundance of Megasphaera (FDR < 0.001), Veillonella $($ FDR $<0.001)$, Rothia $($ FDR $=0.004)$ (Figure 2$)$, Coprobacillus $(\mathrm{FDR}=0.01)$ and Akkermansia $(\mathrm{FDR}=0.008)$ and reduced abundance of Acidaminococcus (FDR $<0.0001)$ and Gemella $(\mathrm{FDR}=0.03)$ (Figure 2).
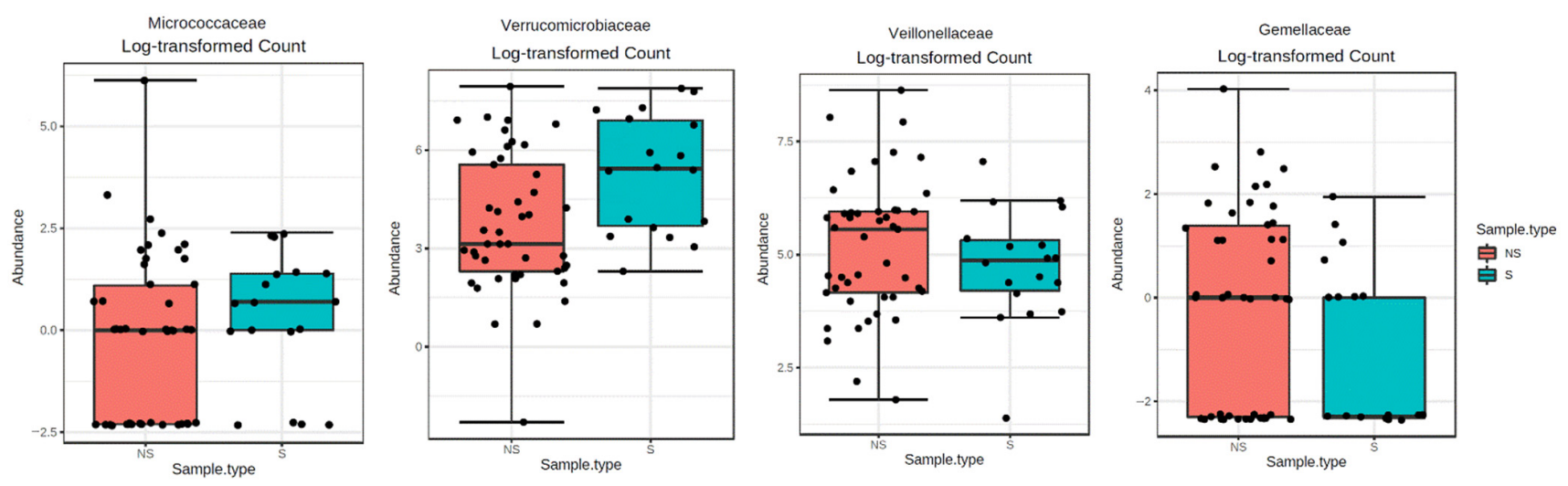

Figure 1. Comparison of statistically significant bacterial families between sarcopenic (S) and not sarcopenic (NS) patients. 

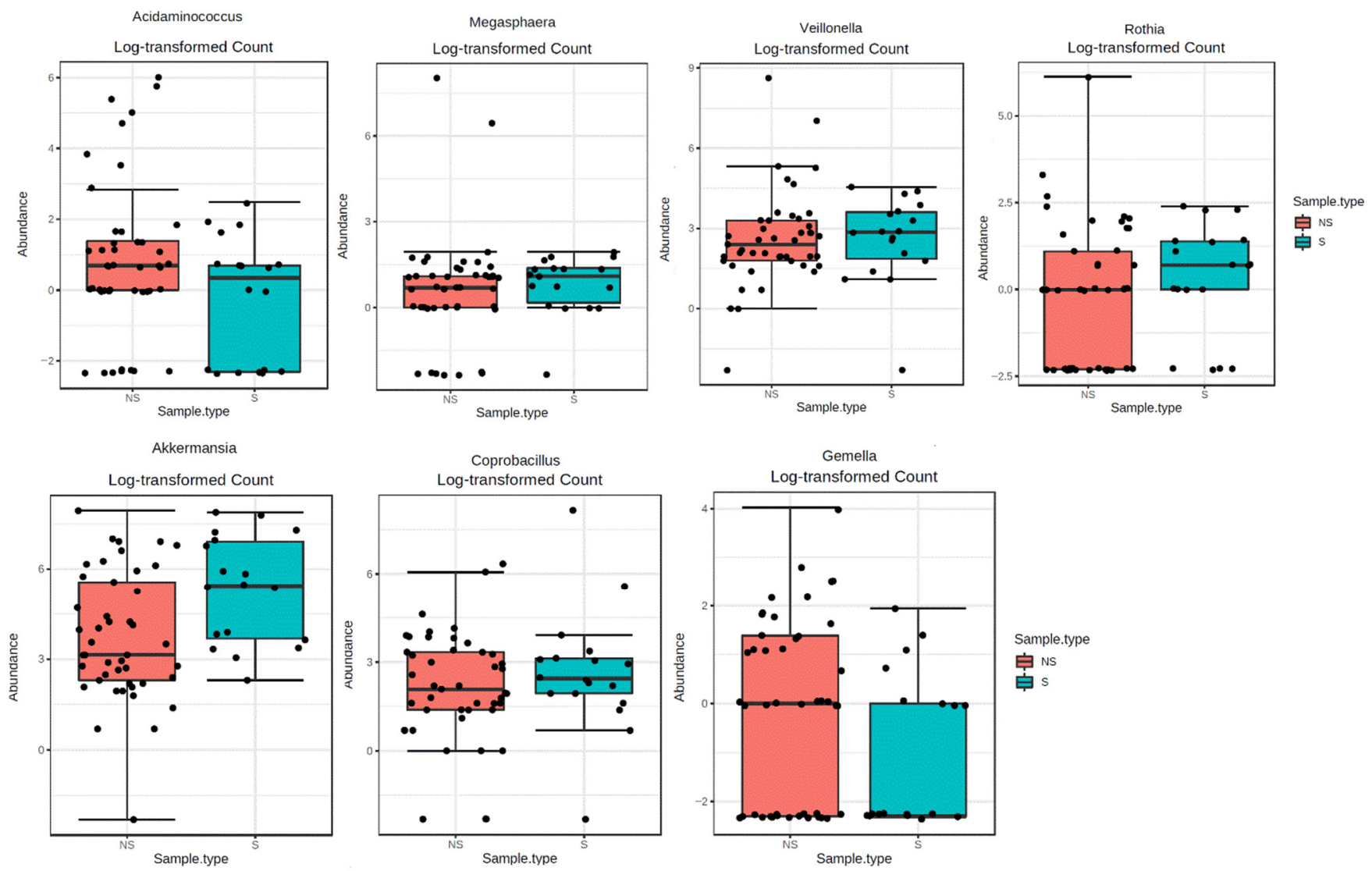

Figure 2. Comparison of statistically significant bacterial genera between sarcopenic (S) and not-sarcopenic (NS) patients.

\subsubsection{Correlations of gMB with Uremic Toxins}

We found a direct correlation between IS and Lactobacillus genus (Table 2), unclassified Lactobacillus and Bifidobacterium longum species (Table 3) and the OTU Bifidobacterium longum $(r=0.28, p=0.02)$ and Bacteroides $183480(r=0,29 ; p=0.024)$ (data not shown).

PCs was directly correlated with four genera-unclassified Coriobacteriaceae, Desulfovibrio (belonging to Proteobacteriaceae family), unclassified Mogibacteriaceae and Christensenellaceae (Table 2)—and two species_Eubacterium biforme and Oscillospira (Table 3). 
Table 2. Association of gMB genera with uremic toxins, oxidative stress and inflammatory cytokines.

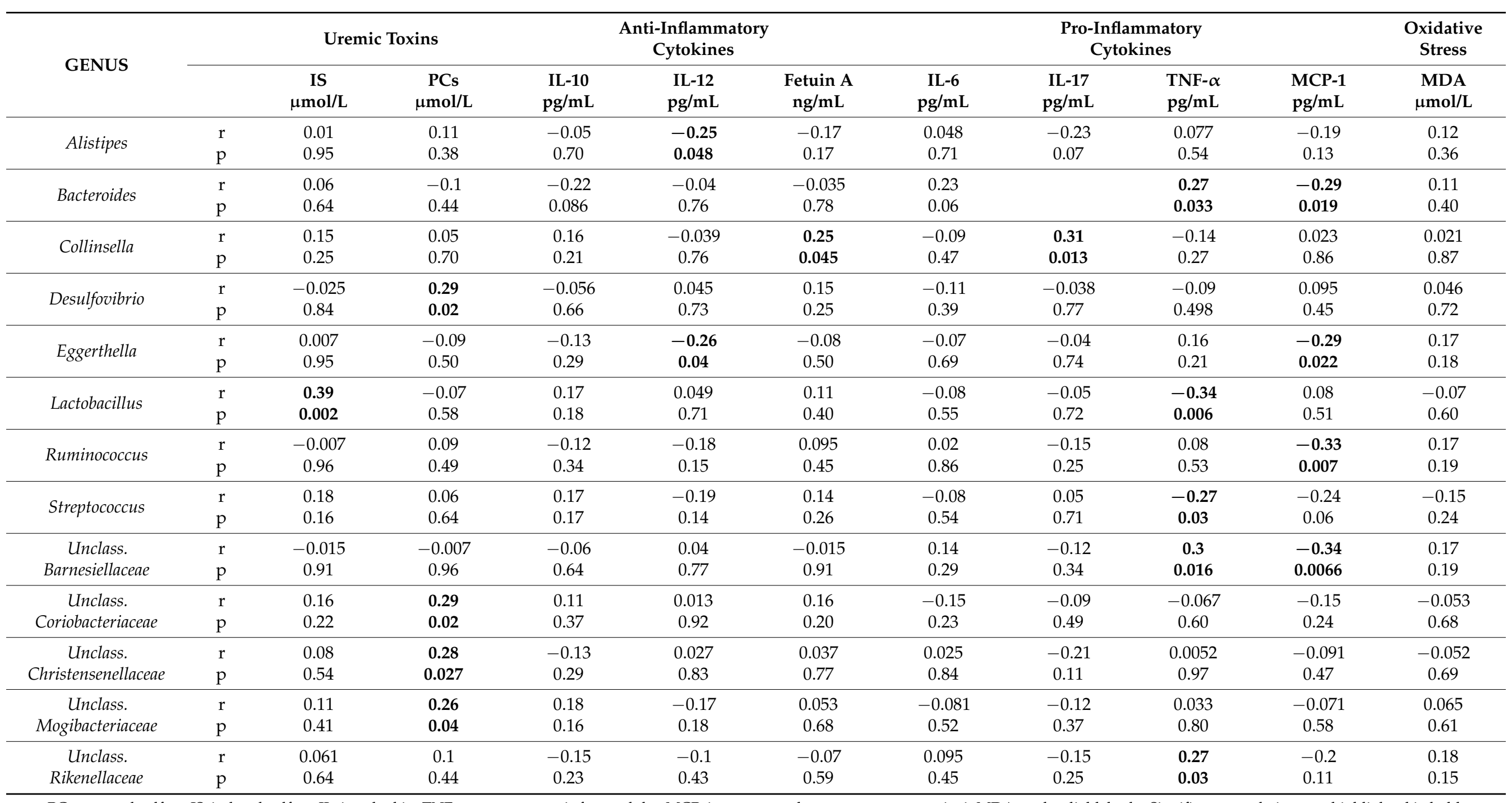

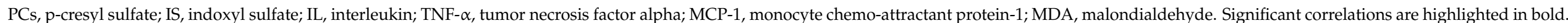


Table 3. Association of gMB species with uremic toxins, oxidative stress and inflammatory cytokines.

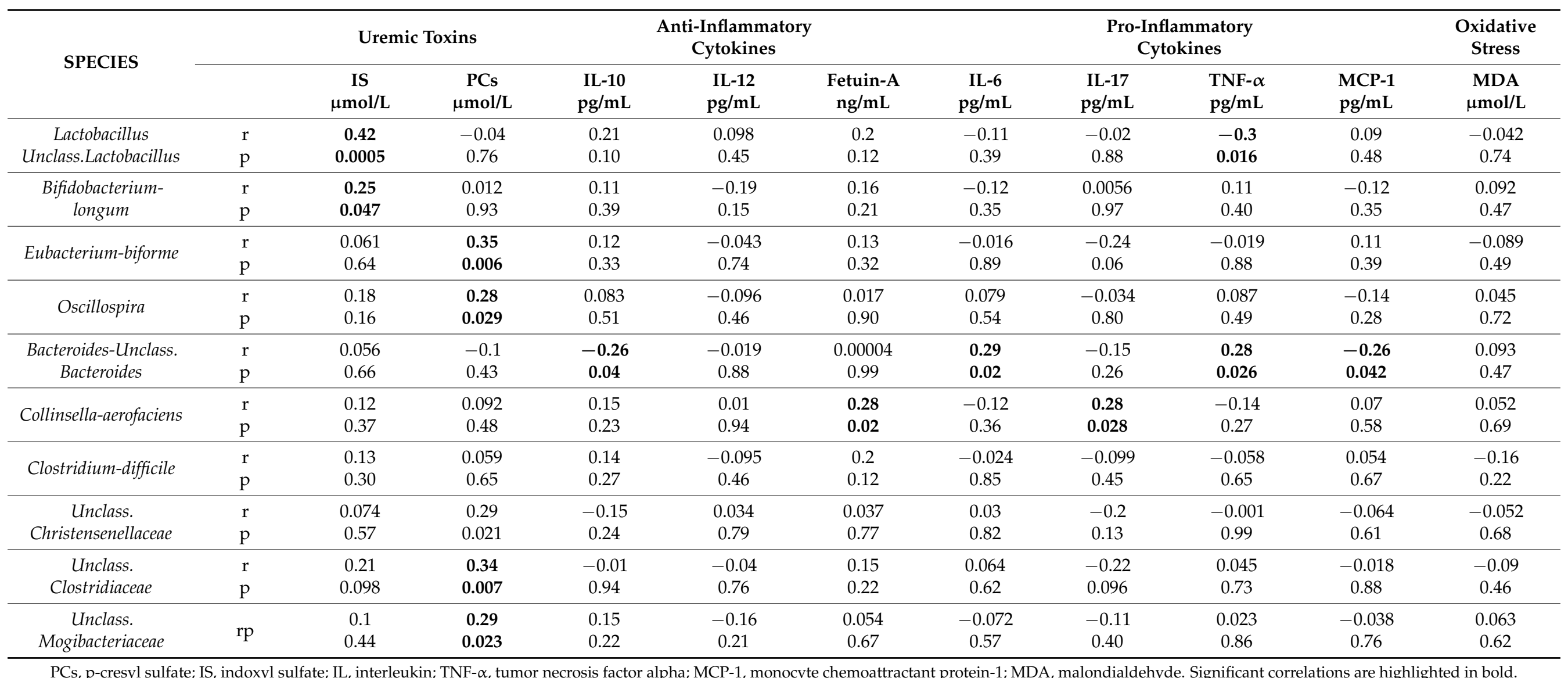




\subsubsection{Correlations of gMB with Inflammatory Cytokines}

Interleukin 10 was inversely associated with unclassified Bacteroides species (Table 4).

Table 4. Comparison of uremic toxins, MDA and cytokines between sarcopenic and not-sarcopenic individuals.

\begin{tabular}{|c|c|c|c|}
\hline Variables & $\begin{array}{l}\text { Sarcopenic } \\
\quad(n=18)\end{array}$ & $\begin{array}{l}\text { Not Sarcopenic } \\
\qquad(n=45)\end{array}$ & $p$ \\
\hline \multicolumn{4}{|c|}{ Uremic toxins } \\
\hline PCs $(\mu \mathrm{mol} / \mathrm{L})$ & $0.65 \pm 0$ & $0.79 \pm 0.18$ & 0.24 \\
\hline IS $(\mu \mathrm{mol} / \mathrm{L})$ & $1.83 \pm 2.61$ & $2.2 \pm 3.13$ & 0.43 \\
\hline \multicolumn{4}{|c|}{ Pro-Inflammatory cytokines } \\
\hline IL-6 (pg/mL) & $2.4 \pm 4.93$ & $2.88 \pm 4.83$ & 0.70 \\
\hline $\mathrm{TNF} \alpha(\mathrm{pg} / \mathrm{mL})$ & $14.63 \pm 8.84$ & $14.14 \pm 8.65$ & 0.99 \\
\hline $\mathrm{MCP}-1(\mathrm{pg} / \mathrm{mL})$ & $428.88 \pm 316.49$ & $447.17 \pm 199.71$ & 0.36 \\
\hline IL17 (pg/mL) & $2.4 \pm 4.93$ & $2.88 \pm 4.83$ & 0.70 \\
\hline \multicolumn{4}{|c|}{ Anti-Inflammatory cytokines } \\
\hline IL12p70 (pg/mL) & $0.605 \pm 1.80$ & $1.471 \pm 2.13$ & 0.45 \\
\hline $\mathrm{IL}-10(\mathrm{pg} / \mathrm{mL})$ & $1.08 \pm 1.66$ & $2.4 \pm 5.87$ & 0.039 \\
\hline Fetuin-A (ng/mL) & $0.56 \pm 0.65$ & $0.64 \pm 0.56$ & 0.56 \\
\hline \multicolumn{4}{|c|}{ Oxidative Stress } \\
\hline $\operatorname{MDA}(\mu \mathrm{mol} / \mathrm{L})$ & $0.275 \pm 0.31$ & $0.346 \pm 0.18$ & 0.47 \\
\hline
\end{tabular}

Interleukin 6 was directly associated with unclassified Bacteroides species (Table 4) and inversely correlated with Bifidobacterium $825808 \mathrm{OTU}(\mathrm{r}=-0.25, p=0.044)$. Interleukin 6 was directly associated only with Bacteroides species (Table 4).

Interleukin 17 was positively associated with the Collinsella genus (Table 3) and particularly with Collinsella aerofaciens species (Table 4 ) and the OTU Collinsella aerofaciens 368175 $(\mathrm{r}=0.3, p=0.019)$.

Tumor necrosis factor alpha was directly associated with some genera-unclassified Rikenellaceae, Bacteroides and unclassified Barnesiellacaeae-and inversely correlated with Streptococcus and Lactobacillus genera (Table 3).

Interleukin 12 was inversely associated with Eggerthella and Alistipes genera (Table 3).

Monocyte chemoattractant protein-1 was negatively associated with unclassified Barnesiellacaeae, Ruminococcus (specifically with Ruminococcus 523140 OTU, $\mathrm{r}=-0.28, p=0.0232$ ), Bacteroides and Eggerthella genera (Table 3).

Fetuin-A was directly associated with the Collinsella genus (Table 3 ) and Collinsella aerofaciens species (specifically with the OTU Collinsella aerofaciens_368175, $\mathrm{r}=0.26, p=0.0371$; Table 3) and negatively associated with Enterobacteriaceae_797229 OTU ( $\mathrm{r}=-0.28, p=0.025)$.

\subsubsection{Correlations of gMB with Oxidative stress}

We found a direct correlation between malondialdehyde and the abundance of three Blautia-related OTUs (Blautia 367790: $\mathrm{r}=0.27, p=0.031$, Blautia 570507: $\mathrm{r}=0.2, p=0.03$, Blautia 316452: $\mathrm{r}=0.26, p=0.038)$ and Ruminococcaceae $361811(\mathrm{r}=0.27, p=0.03)$, and an inverse correlation between MDA and the abundance of the OTU Bacteroides 183480 $(\mathrm{r}=-0.26, p=0.036)$.

\subsection{Sarcopenia, Uremic Toxins, Oxidative Stress and Inflammatory Parameters}

Interleukin 10 concentration was higher in not-sarcopenic than in sarcopenic patients. There were no other differences in the concentrations of IS, PCs, MDA and all the analyzed 
cytokines and inflammatory parameters between sarcopenic and not-sarcopenic patients (Table 4).

\section{Discussion}

The aim of our study was to investigate the associations of the gMB composition with the presence of sarcopenia in older patients with advanced CKD. Secondly, we evaluated if there was any association between the gMB composition and uremic toxins, inflammatory or oxidative stress markers.

Overall twenty-eight\% of our patients were sarcopenic. In this cohort BMI is significantly lower in sarcopenic individuals and this may depend on the correlation between sarcopenia and malnutrition that is quite a common characteristic in older patients with advanced CKD, as it was described also by our group [17].

Consistently with our previous results regarding sarcopenia in older CKD individuals, we did not find any correlation between BMI and inflammatory cytokines.

BMI was, instead, respectively directly and inversely correlated with IS and pCS. These results may at least partially depend on the correlations between poor nutritional status and sarcopenia in our patients. Indeed, in a previous study by our group conducted in older CKD patients, IS and pCS were oppositely associated with nutritional indices [13].

Regarding the gMB composition, we found a greater abundance of the Micrococcaceae and Verrucomicrobiaceae families in sarcopenic compared with not-sarcopenic patients. These families were previously found to be markedly increased in ESRD patients compared with healthy controls [15]. Verrucomicrobiaceae possess tryptophanase (an indole-producing enzyme, leading to increased IS production), while Micrococcaceae possess urease and uricase [18], which, through generation of high quantity of ammonia and ammonium hydroxide, play a key role in the development of uremic enterocolitis [19] and contribute to systemic inflammation [20]. Verrucomicrobia was more abundant in a mice model of chemotherapy-induced malnutrition and muscle-wasting [21]. Higher abundance of Verrucomicrobia was also associated with inflammatory diseases, such as primary sclerosing cholangitis [18,19], psoriasis and psoriatic arthritis [22], and with hepatic encephalopathy in cirrhotic patients, conditions often characterized by protein malnutrition and muscle wasting [23].

Rothia genus was more abundant in the sarcopenic group compared with the nonsarcopenic one. An overrepresentation of Veillonella and Rothia genera has been previously described on the skin of centenarians [24]. These genera have been associated not only with old age, but also with frailty. In frail residents of nursing homes, a shift towards dysbiosis was observed when compared with healthy active community dwellers. In particular, they may have an increased representation of pathobionts such as Veillonella in their salivary microbiota [25] and an increased relative abundance of Rothia mucilaginosa in their nasal microbiota [26]. The presence of these bacteria in salivary microbiota may influence the gMB composition and represent a risk factor for infections, especially pneumonia [27].

In our study, Coprobacillus and Akkermansia were more abundant in sarcopenic patients compared with not-sarcopenic ones.

Coprobacillus has been widely associated not only with biological aging and frailty [28,29] but also with inflammation [30] and hypertensive nephropathy [31].

Akkermansia is considered a beneficial bacterium due to its anti-inflammatory properties, and it has been found to be negatively correlated with inflammatory bowel disease [32], BMI and obesity [33,34], while it is positively associated with weight loss [35]. Akkermansia has been described in great abundance in animal models and in individuals with colorectal carcinomas and multiple sclerosis [28] and it is capable of exacerbating Salmonella typhimurium-induced intestinal inflammation by its ability to disturb host mucus homeostasis [29]. Moreover, some authors reported that the abundance of Akkermansia and other genera increased along with the progression of CKD, suggesting that this bacterium may play an important role in CKD progression [36]. In our study, Akkermansia was more abundant in sarcopenic patients, and it was inversely correlated with BMI (correlation with 
a low statistical strength: $p=0.03, \mathrm{r}=-0.26$ ). These results could possibly influence the inverse relationship linking BMI and sarcopenia [37,38]. However, we do not know if greater abundance of Akkermansia in sarcopenic individuals could also play a pro-inflammatory role influencing the onset of sarcopenia.

In our study, sarcopenic patients were also characterized by lower abundances of the Veillonellaceae and Gemellaceae families as well as of Acidaminococcus and Gemella genera when compared to not-sarcopenic patients. The Gemellaceae and Veillonellaceae families and Gemella genus have been associated with obesity [28,29]. Acidaminococcus species from the Veillonellaceae family may act as opportunistic pathogens [36,39] and can contribute to maintaining a healthy status through the production of short-chain fatty acids (SCFA), such as butyrate and acetate [40]. Moreover, decreased abundance of this genus has been described in CKD patients compared with healthy controls $[14,33]$.

We observed a direct correlation between IS levels and tryptophanase-containing bacteria (Lactobacillus genus, unclassified Lactobacillus and Bifidobacterium longum species and the OTU Bacteroides 183480), which can process tryptophan into indole, which is then metabolized by the liver into IS [41,42]. Previously, we had already found that Lactobacillus abundance was higher in CKD patients compared with healthy controls [43].

We observed also positive correlations between PCs and bacteria that have been associated with several unhealthy conditions. Specifically, the Coriobacteriaceae family comprises many pathobionts involved in human infections, such as Bacteremia and periodontitis; moreover, Coriobacteriaceae family was significantly increased in the ceca of mice in response to stress [44]. In our previous study [43], the Coriobacteriaceae and Mogibacteriaceae families and the Oscillospira genus were more abundant in frail individuals and were positively correlated with markers of inflammation. Oscillibacter-like organisms (including Oscillibacter and Oscillospira) mediate high-fat diet-induced gut dysfunction [45], and Oscillibacter sp. are one of the few bacterial species capable of generating protein-bound uremic toxins, such as IS and PCs, from precursor metabolites under anaerobic conditions [41]. Desulfovibrio belongs to Proteobacteriaceae families that include a wide variety of pathogenic genera involved in diseases, such as cystic fibrosis [42]. Christensenella is generally considered a healthy bacterium [46], while in a large cohort of patients with early kidney disease, Christensenellaceae families were positively associated with IS and PCs levels [47].

Malondialdehyde (MDA) is an advanced oxidation product and a recognized oxidative stress biomarker whose levels are increased in patients with CKD [48]. In our cohort, we found a significant positive correlation between MDA and the presence of four OTUs of the genus Blautia and Ruminococcaceae 361811 and an inverse correlation between MDA and the OTU Bacteroides 183480. An increase of Blautia has been observed in CKD patients [47]. Furthermore Blautia has been previously found to be associated with trimethylamine$\mathrm{N}$-oxide (TMAO) [49] and PCs [50]. Intestinal Bacteroidetes can have a double role in human health: they are known as a major source of propionate but are also involved in the release of toxic products from protein breakdown. Members of this group can exert activities that may help to suppress inflammation, but they also have the potential to promote inflammation and some of them are known to be opportunistic pathogens. In our results, we observed a negative correlation between the presence of OTU Bacteroides 183480, probably a beneficial strain, and MDA. In our study, an unclassified Bacteroides species, which belongs to the Bacteroidetes family, was negatively correlated with interleukin 10 and positively correlated with interleukin 6 , anti-inflammatory and pro-inflammatory cytokines. Interleukin 6 was also negatively correlated with Bifidobacterium 825808 OTU (known to have a probiotic role).

Interleukin 17 was positively correlated with the Collinsella genus and particularly with Collinsella aerofaciens species. The genus Collinsella is the dominant taxon of the family Coriobacteriaceae, commonly considered as pathobionts. Its abundance has been associated with type 2 diabetes [51], rheumatoid arthritis [52] and frailty [43].

We also found a direct correlation between tumor necrosis factor alpha, Bacteroides, unclassified Rikenellaceae and unclassified Barnesiellaceae species. Several species belonging 
to the Rikenellaceae family have been demonstrated to be pathogenic by promoting inflammation and producing mutagenic toxins [53]. Barnesiella is generally considered a healthy bacterium because it prevents pathogenic species of antibiotic-resistant bacteria from colonizing the gut.

Interleukin 12 is inversely correlated with the Eggerthella and Alistipes genera and with Ruminococcus 523140 OTU. All these bacterial genera may have a useful role for human health: Eggerthella lenta is part of the normal human gMB, particularly abundant in frail and old subjects $[15,50]$. In our study, Eggerthella was found to be inversely associated also with monocyte chemoattractant protein-1. Alistipes may have protective effects against some diseases, including liver fibrosis, colitis and cardiovascular disease; however, its role is still debated [54]. Ruminococcus, among other bacteria, belongs to the Clostridium cluster IV, known for their ability to produce butyrate and traditionally considered as healthy bacteria, very important in maintaining intestinal homeostasis [55].

Monocyte chemoattractant protein-1 was found to be inversely associated with unclassified Barnesiellacaeae, Ruminococcus (specifically with Ruminococcus 523140 OTU), Bacteroides and Eggerthella genera. In our previous study, Eggerthella was found to be associated with frailty [43] that may be reasonably considered a surrogate of sarcopenia.

Fetuin A was a directly associated with Collinsella aerofaciens species (and particularly with the OTU Collinsella aerofaciens 368175) and negatively correlated with Enterobacteriaceae 797229 OTU (Tab. 6). Fetuin A is considered as a negative acute phase reactant and its levels are inversely associated with serum CRP [56]. In our study, fetuin A was inversely associated with Enterobacteriaceae 797229 OTU that are considered to be pro-inflammatory. Fetuin-A is also an inhibitor of insulin receptors and its higher levels have been previously associated with insulin resistance $[54,57]$. Insulin resistance is an early metabolic alteration in CKD patients and its prevalence is significantly increased in ESRD. Diabetic patients have higher abundance of Collinsella [58]. Therefore, it may be that the association between Collinsella aerofaciens and fetuin-A that we found in our patients may even influence insulin resistance.

According to our results, none of the correlations observed between gMB, uremic toxins and inflammatory markers seems to be associated with sarcopenia. We did not find any significant difference in blood levels of these markers between the sarcopenic and not-sarcopenic group, except for interleukin 10. However, although interleukin 10 is the only cytokine whose levels are statistically different between sarcopenic and not-sarcopenic individuals, its values do not correlate with any of the bacteria found to be associated with sarcopenia.

Our study has several limitations. Given its cross-sectional design, our results cannot be considered more than descriptive. However, our aim was to explore whether in older CKD patients, there was any difference in the gMB composition between sarcopenic and not-sarcopenic individuals and our results suggest that some differences do exist. The differences in the gMB composition that we found are only slightly significant, but this may depend on the fact that, in order to reduce the possible sources of bias, we applied stringent selection criteria; therefore, the clinical characteristics of our cohort were relatively homogeneous. Advanced age of our patients could make our results not completely representative of all patients with CKD, but we purposely focused our research on older individuals because they have the higher prevalence of sarcopenia.

Finally, the descriptive nature of the study prevents us from discerning if altered gMB is merely the consequence of the inflammatory and toxic milieu characterizing CKD, or if it can actually contribute to the development of sarcopenia in this population.

Overall, we believe that our results may help to formulate new hypotheses to investigate the association between sarcopenia and gut microbiota composition in older patients with advanced CKD. 


\section{Conclusions}

Our results suggest that in elderly patients with advanced CKD, the gMB composition is significantly different between sarcopenic and not-sarcopenic individuals. Furthermore, in CKD patients, there are many mutual correlations between gMB composition, uremic toxins, inflammatory cytokines and oxidative stress parameters.

\section{Materials and Methods}

\subsection{Population Characteristics}

We cross-sectionally evaluated 64 patients affected by stage IIIb-IV CKD $(10<$ eGFR < $45 \mathrm{~mL} / \mathrm{min} / 1.73 \mathrm{~m}^{2}$, not on dialysis), aged $\geq 65$ years, enrolled from those that were consecutively attending the outpatient clinic of the Unit of Nephrology at the Fondazione IRCCS Ca' Granda Ospedale Maggiore Policlinico di Milano. Exclusion criteria were inflammatory diseases and/or ongoing immunosuppressive treatment, cancer, heart failure >NYHA II, decompensated liver disease, use of probiotics/antibiotics within 3 months before study enter and inability to collaborate.

The study was conducted according to the ICP Good Clinical Practices Guidelines and to the Declaration of Helsinki, and it was approved by the Ethics Committee of our Institution (approval number 347/2010, approved on 23 October 2010).

Recruitment of participants started on 1 September 2015 and ended on 6 December 2016. All eligible patients were screened during the observational period and were asked to participate in the study. All participants were requested to sign a written informed consent before being included in the study, as specified in the ICMJE recommendations.

We asked all enrolled patients to complete a questionnaire regarding antibiotics, probiotics and/or any immunosuppressive drugs used within 3 months before fecal sample collection. Each volunteer had to collect feces at home on the day preceding the visit, using $20 \mathrm{~mL}$ plastic sterile stool collection containers, and to place it in their own freezer at $-18 /-20^{\circ} \mathrm{C}$ overnight, before it was stored at $-80^{\circ} \mathrm{C}$ in our laboratories until analysis.

Clinical evaluation and blood samplings were done in the morning after an overnight fasting of at least $10 \mathrm{~h}$.

Sarcopenia was defined according to the criteria of the European Working Group on Sarcopenia in Older People 2 (EWGSOP2) [1].

\subsection{Measurement of Uremic Toxins and Malondialdehyde}

Uremic toxins and malondialdehyde dosing were performed at the laboratory of Biochemistry of the University of Pavia.

Free fraction of indoxyl sulphate and p-cresyl sulphate concentrations in serum were determined by high performance liquid chromatography (HPLC) and fluorescence detection (FLD) [59].

To determine malondialdehyde concentration in serum, we used the HPLC method with fluorescent detection based on the 2-thiobarbituric acid (TBA) assay, using the Chromsystems kit (Chromsystems Instruments \& Chemicals GmbH, 82166, Gräfelfing, Germany) [60].

\subsection{Measurement of Serum Cytokines}

Enzyme-linked immunosorbent assay (ELISA) analyses have been assessed on serum samples at the Laboratory of nephrology of our Institution. Some specific kits were used: Human Interleukin 10 ELISA Kit EHIL10 (Invitrogen, Thermo Fisher Scientific, Monza, Italy), Quantikine ELISA Human CCL2/Monocyte chemoattractant protein-1 Immunoassay DCP00, Quantikine ELISA Human Interleukin 12 p70 Immunoassay D1200 (all R\&D Systems, Space, Milano, Italy) Human tumor necrosis factor alpha ELISA Kit (Thermo Fisher Scientific, Monza, Italy). Quantikine ELISA Human Interleukin 17 Immunoassay, Quantikine HS ELISA Human Interleukin 6 Immunoassay HS600B (R\&D Systems, Space, Milano, Italy). 


\subsection{Bacterial DNA Extraction and V3-V4 Region Sequencing}

Bacterial DNAs were extracted from $50 \mathrm{mg}$ of fecal sample using the FastDNA ${ }^{\mathrm{TM}}$ SPIN Kit for Soil (MP Biomedicals, Lucerna, Switzerland) according to the manufacturer's instructions. The PCR amplifications were performed using the primers $343 \mathrm{~F}$ and $802 \mathrm{R}$. To allow the sequence demultiplex, a specific tag was attached to the forward primer. The PCR amplification and amplicon purification were performed as already described [16].

Sequencing was performed using Illumina's MiSeq platform (Parco Tecnologico Padano, Lodi, Italy) with 300 bp paired-end mode and v3 chemistry and analyzed as previously described. [16]

\subsection{Statistical Analysis}

Results were expressed as mean \pm SD or median \pm IQR. Comparisons of normally distributed variables were done using Student's $t$-test, while the comparisons of not normally distributed ones were done by using Mann-Whitney U test. Proportions and categorical variables were compared by using independent chi-squared $\left(\chi^{2}\right)$ test or Fisher's exact test. Regression analyses were performed by using Pearson or Spearman tests, as appropriate. Statistical analysis was carried out with the Statview software, version 5.0.1.

Sequence analysis were performed using MicrobiomeAnalyst tool $[61,62]$ using the EdgeR algorithm with 0.05 false-discovery rate (FDR) cut-off for identifying significant differences in taxa abundance of bacteria between the two group of subjects.

Statistical significance was set at $p<0.05$.

Supplementary Materials: The following are available online at https:/ /www.mdpi.com/article/10 $.3390 /$ toxins13070472/s1, Figure S1: Distribution of bacterial families, expressed as relative abundance, in samples of sarcopenic (S) and non-sarcopenic (NS) subjects., Figure S2: Distribution of bacterial genera, expressed as relative abundance, in samples of sarcopenic (S) and not sarcopenic (NS) subjects., Table S1: Correlation between bacterial familiae and clinical and biochemical variables, Table S2: Correlation between genus and clinical and biochemical variables.

Author Contributions: Conceptualization, E.M., P.M. and M.L.C.; methodology, M.L.C., F.M., S.A. and V.R.; software, F.Z. and L.C.; validation, P.M. and S.V.; formal analysis, L.C. and M.L.C.; investigation, E.M., L.C., M.L.C., F.M., S.A. and V.R.; resources, P.M.; data curation, F.Z. and L.C.; writing—original draft preparation, E.M. and L.C.; writing—review and editing, F.Z. and S.V.; visualization, L.C. and F.M.; supervision, S.V.; project administration, S.V.; funding acquisition, P.M. All authors have read and agreed to the published version of the manuscript.

Funding: This research received no external funding.

Institutional Review Board Statement: The study was conducted according to the guidelines of the Declaration of Helsinki and approved by the Ethics Committee of our Institution (Comitato Etico Milano Area 2 (approval document 347/2010, PROVE: PROteinuria and Vascular End points). Informed consent was obtained from all subjects involved in the study.

Informed Consent Statement: Informed consent was obtained from all subjects involved in the study.

Data Availability Statement: The data presented in this study are openly available in Dryad and FlowRepository at doi:10.1371/journal.pone.0228530 [16].

Conflicts of Interest: The authors declare no conflict of interest.

\section{References}

1. Cruz-Jentoft, A.J.; Bahat, G.; Bauer, J.; Boirie, Y.; Bruyère, O.; Cederholm, T.; Cooper, C.; Landi, F.; Rolland, Y.; Sayer, A.A.; et al. Sarcopenia: Revised European consensus on definition and diagnosis. Age Ageing 2019, 48, 601. [CrossRef] [PubMed]

2. Watanabe, H.; Enoki, Y.; Maruyama, T. Sarcopenia in chronic kidney disease: Factors, mechanisms, and therapeutic interventions. Biol. Pharm. Bull. 2019, 42, 1437-1445. [CrossRef]

3. Nataatmadja, M.; Cho, Y.; Campbell, K.; Johnson, D.W. The roles of indoxyl sulphate and p-cresyl sulphate in patients with chronic kidney disease: A review of therapeutic options. In Chronic Kidney Disease-From Pathophysiology to Clinical Improvements; Rath, T., Ed.; InTech: London, UK, 2018. 
4. Mair, R.D.; Sirich, T.L.; Plummer, N.S.; Meyer, T.W. Characteristics of colon-derived uremic solutes. Clin. J. Am. Soc. Nephrol. 2018, 13, 1398-1404. [CrossRef] [PubMed]

5. Evenepoel, P.; Meijers, B.; Bammens, B.; Verbeke, K. Uremic toxins originating from colonic microbial metabolism. Kidney Int. 2009, 76, S12-S19. [CrossRef] [PubMed]

6. Barreto, F.C.; Barreto, D.V.; Liabeuf, S.; Meert, N.; Glorieux, G.; Temmar, M.; Choukroun, G.; Vanholder, R.; Massy, Z.A.; European Uremic Toxin Work Group (EUTox). Serum indoxyl sulfate is associated with vascular disease and mortality in chronic kidney disease patients. Clin. J. Am. Soc. Nephrol. 2009, 4, 1551-1558. [CrossRef] [PubMed]

7. Koppe, L.; Pillon, N.; Vella, R.E.; Croze, M.L.; Pelletier, C.C.; Chambert, S.; Massy, Z.; Glorieux, G.; Vanholder, R.; Dugenet, Y.; et al. P-cresyl sulfate promotes insulin resistance associated with CKD. J. Am. Soc. Nephrol. 2012, 24, 88-99. [CrossRef] [PubMed]

8. Liabeuf, S.; Barreto, D.V.; Barreto, F.C.; Meert, N.; Glorieux, G.; Schepers, E.; Temmar, M.; Choukroun, G.; Vanholder, R.; Massy, Z.A.; et al. Free $p$-cresyl sulphate is a predictor of mortality in patients at different stages of chronic kidney disease. Nephrol. Dial. Transplant. 2009, 25, 1183-1191. [CrossRef]

9. Yamamoto, H.; Tsuruoka, S.; Ioka, T.; Ando, H.; Ito, C.; Akimoto, T.; Fujimura, A.; Asano, Y.; Kusano, E. Indoxyl sulfate stimulates proliferation of rat vascular smooth muscle cells. Kidney Int. 2006, 69, 1780-1785. [CrossRef]

10. Adijiang, A.; Goto, S.; Uramoto, S.; Nishijima, F.; Niwa, T. Indoxyl sulphate promotes aortic calcification with expression of osteoblast-specific proteins in hypertensive rats. Nephrol. Dial. Transplant. 2008, 23, 1892-1901. [CrossRef]

11. Sato, E.; Mori, T.; Mishima, E.; Suzuki, A.; Sugawara, S.; Kurasawa, N.; Saigusa, D.; Miura, D.; Morikawa-Ichinose, T.; Saito, R.; et al. Metabolic alterations by indoxyl sulfate in skeletal muscle induce uremic sarcopenia in chronic kidney disease. Sci. Rep. 2016, 6, 36618. [CrossRef]

12. Changchien, C.-Y.; Lin, Y.-H.; Cheng, Y.-C.; Chang, H.-H.; Peng, Y.-S.; Chen, Y. Indoxyl sulfate induces myotube atrophy by ROS-ERK and JNK-MAFbx cascades. Chem. Interact. 2019, 304, 43-51. [CrossRef] [PubMed]

13. Caldiroli, L.; Armelloni, S.; Eskander, A.; Messa, P.; Rizzo, V.; Margiotta, E.; Cesari, M.; Vettoretti, S. Association between the uremic toxins indoxyl-sulfate and $p$-cresyl-sulfate with sarcopenia and malnutrition in elderly patients with advanced chronic kidney disease. Exp. Gerontol. 2021, 147, 111266. [CrossRef]

14. Briskey, D.; Tucker, P.; Johnson, D.W.; Coombes, J. The role of the gastrointestinal tract and microbiota on uremic toxins and chronic kidney disease development. Clin. Exp. Nephrol. 2017, 21, 7-15. [CrossRef] [PubMed]

15. Andersen, K.; Kesper, M.S.; Marschner, J.A.; Konrad, L.; Ryu, M.; Vr, S.K.; Kulkarni, O.P.; Mulay, S.R.; Romoli, S.; Dem-leitner, J.; et al. Intestinal dysbiosis, barrier dysfunction, and bacterial translocation account for CKD-related systemic in-flammation. J. Am. Soc. Nephrol. 2017, 28, 76-83. [CrossRef] [PubMed]

16. Margiotta, E.; Miragoli, F.; Callegari, M.L.; Vettoretti, S.; Caldiroli, L.; Meneghini, M.; Zanoni, F.; Messa, P. Gut microbiota composition and frailty in elderly patients with chronic kidney disease. PLoS ONE 2020, 15, e0228530. [CrossRef] [PubMed]

17. Vettoretti, S.; Caldiroli, L.; Armelloni, S.; Ferrari, C.; Cesari, M.; Messa, P. Sarcopenia is associated with malnutrition but not with systemic inflammation in older persons with advanced CKD. Nutrients 2019, 11, 1378. [CrossRef]

18. Ntemiri, A. Effect of Dairy Based Food Ingredients on the Gut Microbiota of Older Consumers. Ph.D. Thesis, University College Cork, Cork, Ireland, 2019.

19. Marchesi, J.R.; Adams, D.H.; Fava, F.; Hermes, G.D.A.; Hirschfield, G.M.; Hold, G.; Quraishi, M.N.; Kinross, J.; Smidt, H.; Tuohy, K.M.; et al. The gut microbiota and host health: A new clinical frontier. Gut 2016, 65, 330-339. [CrossRef]

20. Myers, B.; Brownstone, N.; Reddy, V.; Chan, S.; Thibodeaux, Q.; Truong, A.; Bhutani, T.; Chang, H.-W.; Liao, W. The gut microbiome in psoriasis and psoriatic arthritis. Best Pract. Res. Clin. Rheumatol. 2019, 33, 101494. [CrossRef]

21. Merli, M.; Iebba, V.; Giusto, M. What is new about diet in hepatic encephalopathy. Metab. Brain Dis. 2016, 31, 1289-1294. [CrossRef]

22. Yang, X.; Feng, H.; Zhan, X.; Zhang, C.; Cui, R.; Zhong, L.; Ying, S.; Chen, Z. Early-life vancomycin treatment promotes airway inflammation and impairs microbiome homeostasis. Aging 2019, 11, 2071-2081. [CrossRef]

23. Biagi, E.; Nylund, L.; Candela, M.; Ostan, R.; Bucci, L.; Pini, E.; Nikkila, J.; Monti, D.; Satokari, R.; Franceschi, C.; et al. Through ageing, and beyond: Gut microbiota and inflammatory status in seniors and centenarians. PLoS ONE 2010, 5, e10667. [CrossRef]

24. Kaczmarek, J.L.; Musaad, S.M.; Holscher, H.D. Time of day and eating behaviors are associated with the composition and function of the human gastrointestinal microbiota. Am. J. Clin. Nutr. 2017, 106, ajcn156380. [CrossRef] [PubMed]

25. Jackson, M.A.; Jeffery, I.B.; Beaumont, M.; Bell, J.T.; Clark, A.G.; Ley, R.E.; O’Toole, P.W.; Spector, T.D.; Steves, C.J. Signatures of early frailty in the gut microbiota. Genome Med. 2016, 8, 1-11. [CrossRef]

26. Maffei, V.J.; Kim, S.; Blanchard, E.; Luo, M.; Jazwinski, S.M.; Taylor, C.; Welsh, D.A. Biological aging and the human gut microbiota. J. Gerontol. Ser. A Biomed. Sci. Med. Sci. 2017, 72, 1474-1482. [CrossRef] [PubMed]

27. Terzo, S.; Mulè, F.; Caldara, G.F.; Baldassano, S.; Puleio, R.; Vitale, M.; Cassata, G.; Ferrantelli, V.; Amato, A. Pistachio consumption alleviates inflammation and improves gut microbiota composition in mice fed a high-fat diet. Int. J. Mol. Sci. 2020, $21,365$. [CrossRef]

28. Bianchi, L.; Abete, P.; Bellelli, G.; Bo, M.; Cherubini, A.; Corica, F.; Di Bari, M.; Maggio, M.; Manca, G.M.; Rizzo, M.R.; et al. Prevalence and clinical correlates of sarcopenia, identified according to the EWGSOP definition and diagnostic algorithm, in hospitalized older people: The GLISTEN study. J. Gerontol. Ser. A Biomed. Sci. Med. Sci. 2017, 72, 1575-1581. [CrossRef]

29. Peters, B.A.; Shapiro, J.A.; Church, T.R.; Miller, G.; Trinh-Shevrin, C.; Yuen, E.; Friedlander, C.; Hayes, R.B.; Ahn, J. A taxonomic signature of obesity in a large study of American adults. Sci. Rep. 2018, 8, 1-13. [CrossRef] 
30. Barlow, G.M.; Lin, E.A.; Mathur, R. An overview of the roles of the gut microbiome in obesity and diabetes. In Nutritional and Therapeutic Interventions for Diabetes and Metabolic Syndrome; Bagchi, D., Ed.; Elsevier: London, UK, 2018; pp. 65-91.

31. Dhakal, S.; McCormack, L.; Dey, M. Association of the gut microbiota with weight-loss response within a retail weightmanagement program. Microorganisms 2020, 8, 1246. [CrossRef]

32. Freedman, S.N.; Shahi, S.K.; Mangalam, A.K. The "gut feeling": Breaking down the role of gut microbiome in multiple sclerosis. Neurotherapeutics 2018, 15, 109-125. [CrossRef]

33. Rogosa, M. Acidaminococcus gen. n., Acidaminococcus fermentans sp. n., anaerobic gram-negative diplococci using amino acids as the sole energy source for growth. J. Bacteriol. 1969, 98. [CrossRef]

34. Hu, Q.; Wu, K.; Pan, W.; Zeng, Y.; Hu, K.; Chen, D.; Huang, X.; Zhang, Q. Intestinal flora alterations in patients with early chronic kidney disease: A case-control study among the Han population in southwestern China. J. Int. Med. Res. 2020, 48. [CrossRef] [PubMed]

35. Zhou, Q.; Zhang, Y.; Wang, X.; Yang, R.; Zhu, X.; Zhang, Y.; Chen, C.; Yuan, H.; Yang, Z.; Sun, L. Gut bacteria Akkermansia is associated with reduced risk of obesity: Evidence from the American gut project. Nutr. Metab. 2020, 17, 1-9. [CrossRef] [PubMed]

36. Pisanu, S.; Palmas, V.; Madau, V.; Casula, E.; Deledda, A.; Cusano, R.; Uva, P.; Vascellari, S.; Boi, F.; Loviselli, A.; et al. Impact of a moderately hypocaloric Mediterranean diet on the gut microbiota composition of Italian obese patients. Nutrients 2020, $12,2707$. [CrossRef] [PubMed]

37. Vaziri, N.D.; Wong, J.; Pahl, M.; Piceno, Y.; Yuan, J.; DeSantis, T.Z.; Ni, Z.; Nguyen, T.-H.; Andersen, G.L. Chronic kidney disease alters intestinal microbial flora. Kidney Int. 2013, 83, 308-315. [CrossRef] [PubMed]

38. Gryp, T.; Huys, G.R.; Joossens, M.; Van Biesen, W.; Glorieux, G.; Vaneechoutte, M. Isolation and quantification of uremic toxin precursor-generating gut bacteria in chronic kidney disease patients. Int. J. Mol. Sci. 2020, 21, 1986. [CrossRef]

39. Brook, I. Anaerobic infections in childhood. Clin. Infect. Dis. 1984, 6, S187-S192. [CrossRef]

40. Zhang, L.S.; Davies, S.S. Microbial metabolism of dietary components to bioactive metabolites: Opportunities for new therapeutic interventions. Genome Med. 2016, 8, 1-18. [CrossRef]

41. Barrios, C.; Beaumont, M.; Pallister, T.; Villar, J.; Goodrich, J.K.; Clark, A.; Pascual, J.; Ley, R.; Spector, T.D.; Bell, J.T.; et al. Gut-microbiota-metabolite axis in early renal function decline. PLoS ONE 2015, 10, e0134311. [CrossRef]

42. Terrier-Lenglet, A.; Nollet, A.; Liabeuf, S.; Barreto, D.V.; Brazier, M.; Lemke, H.D.; Vanholder, R.; Choukroun, G.; Massy, Z.A.; Groupe EUTox (European Uremic toxin). Plasma malondialdehyde may not predict mortality in patient with chronic kidney disease. Nephrol. Ther. 2011, 7, 219-224. [CrossRef]

43. Xu, K.-Y.; Xia, G.-H.; Lu, J.-Q.; Chen, M.-X.; Zhen, X.; Wang, S.; You, C.; Nie, J.; Zhou, H.-W.; Yin, J. Impaired renal function and dysbiosis of gut microbiota contribute to increased trimethylamine-N-oxide in chronic kidney disease patients. Sci. Rep. 2017, 7, 1-12. [CrossRef]

44. Schippa, S.; Iebba, V.; Santangelo, F.; Gagliardi, A.; De Biase, R.V.; Stamato, A.; Bertasi, S.; Lucarelli, M.; Conte, M.P.; Quattrucci, S.; et al. Cystic fibrosis transmembrane conductance regulator (CFTR) allelic variants relate to shifts in faecal microbiota of cystic fibrosis patients. PLoS ONE 2013, 8, e61176. [CrossRef]

45. Waters, J.L.; Ley, R.E. The human gut bacteria Christensenellaceae are widespread, heritable, and associated with health. BMC Biol. 2019, 17, 1-11. [CrossRef]

46. Feng, Y.-L.; Cao, G.; Chen, D.-Q.; Vaziri, N.D.; Chen, L.; Zhang, J.; Wang, M.; Guo, Y.; Zhao, Y.-Y. Microbiome-metabolomics reveals gut microbiota associated with glycine-conjugated metabolites and polyamine metabolism in chronic kidney disease. Cell. Mol. Life Sci. 2019, 76, 4961-4978. [CrossRef]

47. Saito, Y.; Sato, T.; Nomoto, K.; Tsuji, H. Identification of phenol- and $p$-cresol-producing intestinal bacteria by using media supplemented with tyrosine and its metabolites. FEMS Microbiol. Ecol. 2018, 76, 4961-4978. [CrossRef]

48. Carson, T.; Lowe, J.; Ramaraj, T.; Leff, J.W.; Luo, L.; Bell, C.J.; Shah, V.O. Composition diversity and abundance of gut microbiome in prediabetes and type 2 diabetes. J. Diabetes Obes. 2015, 2, 1-7.

49. Chen, J.; Wright, K.; Davis, J.M.; Jeraldo, P.; Marietta, E.V.; Murray, J.; Nelson, H.; Matteson, E.L.; Taneja, V. An expansion of rare lineage intestinal microbes characterizes rheumatoid arthritis. Genome Med. 2016, 8, 1-14. [CrossRef]

50. Sun, T.; Liu, S.; Zhou, Y.; Yao, Z.; Zhang, D.; Cao, S.; Wei, Z.; Tan, B.; Li, Y.; Lian, Z.; et al. Evolutionary biologic changes of gut microbiota in an "adenoma-carcinoma sequence" mouse colorectal cancer model induced by 1, 2-Dimethylhydrazine. Oncotarget 2016, 8, 444-457. [CrossRef]

51. Ye, J.; Lv, L.; Wu, W.; Li, Y.; Shi, D.; Fang, D.; Guo, F.; Jiang, H.; Yan, R.; Ye, W.; et al. Butyrate protects mice against methioninecholine-deficient diet-induced non-alcoholic steatohepatitis by improving gut barrier function, attenuating inflammation and reducing endotoxin levels. Front. Microbiol. 2018, 9, 1967. [CrossRef] [PubMed]

52. Masood, M.I.; Qadir, M.I.; Shirazi, J.H.; Khan, I.U. Beneficial effects of lactic acid bacteria on human beings. Crit. Rev. Microbiol. 2010, 37, 91-98. [CrossRef] [PubMed]

53. Biagi, E.; Franceschi, C.; Rampelli, S.; Severgnini, M.; Ostan, R.; Turroni, S.; Consolandi, C.; Quercia, S.; Scurti, M.; Monti, D.; et al. Gut microbiota and extreme longevity. Curr. Biol. 2016, 26, 1480-1485. [CrossRef]

54. Vörös, K.; Szenthe, P.; Kaszás, E.; Cseh, K.; Gráf, L., Jr.; Prohászka, Z.; Gráf, L.; Böröcz, Z.; Kalabay, L. Serum fetuin-A in metabolic and inflammatory pathways in patients with myocardial infarction. Eur. J. Clin. Investig. 2011, 41, 703-709. [CrossRef] [PubMed]

55. Laughlin, G.A.; Barrett-Connor, E.; Cummins, K.M.; Daniels, L.B.; Wassel, C.L.; Ix, J.H. Sex-specific association of fetuin-a with type 2 diabetes in older community-dwelling adults: The Rancho Bernardo study. Diabetes Care 2013, 36, 1994-2000. [CrossRef] 
56. Zhang, X.; Shen, D.; Fang, Z.; Jie, Z.; Qiu, X.; Zhang, C.; Chen, Y.; Ji, L. Human gut microbiota changes reveal the progression of glucose intolerance. PLoS ONE 2013, 8, e71108. [CrossRef] [PubMed]

57. Ix, J.H.; Wassel, C.L.; Kanaya, A.M.; Vittinghoff, E.; Johnson, K.C.; Koster, A.; Cauley, J.A.; Harris, T.B.; Cummings, S.R.; Shlipak, M.J. Fetuin-A and incident diabetes mellitus in older persons. JAMA 2008, 300, 182-188. [CrossRef] [PubMed]

58. Lee, Y.-T.N. Urea concentration in intestinal fluids in normal and uremic dogs. J. Surg. Oncol. 1971, 3, 163-168. [CrossRef]

59. Pretorius, C.J.; McWhinney, B.C.; Sipinkoski, B.; Johnson, L.A.; Rossi, M.; Campbell, K.L.; Ungerer, J.P. Reference ranges and biological variation of free and total serum indoxyl- and $p$-cresyl sulphate measured with a rapid UPLC fluorescence detection method. Clin. Chim. Acta 2013, 419, 122-126. [CrossRef]

60. Grune, T.; Siems, W.; Esterbauer, H. 077 comparison of different assays for malondialdehyde using thiobarbituric acid. Anal. Bioanal. Chem. 1992, 343, 135. [CrossRef]

61. Chong, J.; Liu, P.; Zhou, G.; Xia, J. Using MicrobiomeAnalyst for comprehensive statistical, functional, and meta-analysis of microbiome data. Nat. Protoc. 2020, 15, 799-821. [CrossRef]

62. Dhariwal, A.; Chong, J.; Habib, S.; King, I.L.; Agellon, L.B.; Xia, J. MicrobiomeAnalyst: A web-based tool for comprehensive statistical, visual and meta-analysis of microbiome data. Nucleic Acids Res. 2017, 45, W180-W188. [CrossRef] 